\title{
Administration via Implantation
}

National Cancer Institute

\section{Source}

National Cancer Institute. Administration via Implantation. NCI Thesaurus. Code C38201.

Drug administration via imbedded implants used as depot formulations to limit high drug concentrations to the area surrounding the pathology or to provide sustained drug release for systemic therapy. Implant systems are used when chronic therapy is indicated and may take the form of highly viscous liquids, semi-solid formulations, rod impregnated with drug substances, or a liquid which gels in situ. Release kinetics may be controlled by varying polymer composition, different types of stimuli, such as electrical, and varying of implant structure, e.g. as coating. 